\title{
EFFECT OF SOME ESSENTIAL OILS ON CONTROLLING SUGAR BEET DAMPING - OFF AND ROOT ROT DISEASES CAUSED BY MANY SERIOUS SOIL BORNE FUNGAL PATHOGENS IN NILE DELTA REGION . \\ Gouda, M.I.* ; Samia A. El-Fahar ${ }^{\star *}$ and Fathia S. El-Shoraky* \\ * Plant Pathology Res. Institute, Agric. Research Center, Giza, Egypt. \\ ${ }^{*}$ Sugar Crops Research Institute, Agric. Research Center, Giza, Egypt
}

\begin{abstract}
Testing the effect of some essenstial oils on controlling of some causal pathogens of sugar beet plants in Nile Delta soil,( Sclerotium rolfsii Sacc.; Rhizoctonia solani Khun.; Fusarium oxysporum Schlech and Macrophomina phaseolina) (Tassi) Goid. In vitro, oils of Syzygium aromaticum L. (clove), Cuminum cyminum L. (cumin), Ocimum basilicum L.(basil) Mentha viridis (mint) were effective as inhibitors of the linear growth of $S$. rolfsii $R$. solani, $M$. Phaseolina at the concentration of $1500 \mathrm{ppm}$. On the other hand, they were affected slightly the linear growth and sporulation of $F$. Oxysporum at the concentration of $2000 \mathrm{ppm}$. At the same time, increasing the concentration of the tested materials significantly reduced the linear growth of all the tested pathogens.

Under greenhouse and field conditions, data show that oils have significant effect in improving the number of survived seedlings and reduce of root rot. Oils of Syzygium aromaticum L. (Clove) and Cuminum syminum L. (Cumin) were suprior than the other materials in reducing infection with pre- and post damping - off and root rots of sugar beet as well as the disease severity. The rest oils were less effective in decreasing the disease incidence. Oils improved morpholigical characters expressed as plant height, Leaf area and dry weight per plant, also increasing the yield component, total soluble sugars (TSS), sucrose percent in root and sugar purity.

The fungicide Rizolex T. 50 was used for comparative studies in controlling these diseases.
\end{abstract}

Keywords: Sugar beet, Root rot diseases, Soil borne pathogens (S. rolfsii, R. solani, F. oxysporum and M. phaseolina ), Essential oils.

\section{INTRODUCTION}

Sugar beet (Beta vulgaris L.) has become recently one of the most economically important crops in Egypt. This crop is liable to be attacked by certain soil-borne pathogens at all stages of growth causing pre-and post emergance damping-off, as well as various degrees of root-rots. Sclerotium rolfsii and Rhizoctonia solani were considered among the most destructive diseases affecting yield crop in Egypt (El-Abyad et al., 1992; El-Kazzaz et al.,1999 and El- Kholi 2000 ; Eah 2000 and Gouda 2001). Some essential oils have an allelopathic effect on some diseases on other plant hosts as previous investigators have reported (Jain,et al.,1992; Paran et al.,1996; Elshoraky 1998 and Gouda, 2001).

The present work aimed to study the effect of certain plant oils on controling sugar beet root rot disease caused by important soil-borne fungal pathogens in Nile Delta region 
Gouda, M.l. et al.

\section{MATERIALS AND METHODS}

Essential oils :The following essential oils used in this study were :Ocimum basilicum L.(Basil), Syzygium aromaticum L. (Clove), Mentha viridis (Mint), Cuminum syminum L. (Cumin).They were purchased from Ghomhoriya Comp. for Medicine and Chemicals

In vitro experiment :Essential oils were incorporated into melted PDA madium just before solidification at the tested concentrations (1000, 1500 and $2000 \mathrm{ppm}$ ) and poured into Petri dishes $(9 \mathrm{~cm}$ in diameter). Plates were inoculated at the center with $5 \mathrm{~mm}$-culture discs of fungi under study and incubated at $28 \stackrel{\circ}{ } \mathrm{C}$. Radial growth of each fungus was determined daily by measuring colony diameter in each of four replicate plates. Percentage of reduction in colony diameter was calculated for each treatment.

Sporulations of $F$. oxysporum was also determined after 15 days of incubation. Spores were collected from each dish by gentle brushing of the colony surface and collecting spores were suspended in $10 \mathrm{ml}$ of sterilized distilled water. The collected spore suspension was then sieved through cheese cloth to remove the mycelial fragments. Spore suspensions were resuspended into sterile water to give a final volume of $100 \mathrm{ml}$. Spore concentration (No. of spores $/ \mathrm{ml}$ ) in each treatment was calculated by the aid of a haemocytometer (Mandeel \& Backer, 1991 ).

Greenhouse experiments : Essential oils were evaluated for their efficiency against damping- off and root-rot diseases caused by $S$. rolfsii and $R$. solani under greenhouse conditions. Seeds of sugar beet Kawmera C.v were dressed into a concentration of $2000 \mathrm{ppm}$ of each of the oils under study for 8 hours before planting. Sugar beet seeds soaked only in water for 8 hrs served as a check treatment. Seeds were treated with Rhizolex T-50 at the rate recommended dose $(2.5 \mathrm{Kg})$ and seed were cultivated in $R$. solani and S. rolfsii - infested soil (15 seeds / pot). Three replicate pots (No.35) were used and uninfested soil acted as control. Disease readings were taken 15 , $45 \& 150$ days after planting for pre \& post emergence damping - off and root rot respectively. Root yield per plant and yield losses due to infection were also estimated at harvest time (150 days of planting). Yield component i.e. total soluble solids (TSS), sucrose percent and sugar purity were also estimated. TSS was estimated in fresh roots using the hand refractometer according to McGinnis (1982). Sucrose percent was estimated according to (A.O.A.C.,1990) by adding $173 \mathrm{ml} 3 \%$ lead acetate to $26 \mathrm{~g}$ from the sample representing the interior of the roots. After filteration, sucrose percent was measured by the aid of sacarometer. Purity percent was calculated by dividing the sucrose percent by TSS. Also plant height, leaf area and leaf dry weight were estimated after 150 days of planting. Leaf area (mm2) was determined using LI-3100 area meter according to Aly et. al.(1996).

Field experiments: Experiments were carried out to study the effect of some oils on root rot incidence and yield per plot was also studied. These experiments were performed in the farm of Sakha in two successive seasons i.e. 2005 - 2006 and 2006 - 2007. Randomized complete blocks design with three replicate plots (1/400 feddan) was used. Pre and post emergence 
damping - off were taken after 15 and 45 days of planting respectively. Root rots were estimated and recorded along with the yield per plot at harvest time (about 200 days of planting). Disease readings were taken and recorded as percentage of infection and disease severity at harvest (Sharma, and PathaK 1994).

Statistical analysis : Duncan's multiple range test (DMRT). Analysis was performed by the software A micro computer programme for the design, Management and Analysis of Agronomic Research Experiments Averages were compared to the least significant difference (LSD) and (Irristat Michigan state Univ., USA,1993).

\section{RESULTS}

In vitro experiments :Oils prepared as mentioned under Materials \& Methods were examined for their effect on the linear growth of some soilborne fungal pathogen in Petri dishes.

Results shown in Tables (1, 2, 3 and 4) indicate that all experimented materials were positively effective in reducing the linear growth of soil borne fungal pathogens, generally. The effect was obviously increased by increasing the concentration of oils from 1000 to $2000 \mathrm{ppm}$. The obtained datd in Table (1) show that oils of each of $S$. aromaticum (clove), C. cyminum (cumin), O. basilicum (basil) \& M. viridis (mint) were effective as inhibitors to growth of $S$. rolfsii. All of these oils except, the mint oil were strongly inhibited the growth of the pathogen at the concentration of $1000 \mathrm{ppm}$.

All oils under study significantly retarded the linear growth of $R$. solani and M. Phaseolina, on PDA at 1500 ppm. Tables (2 \& 4).On the other hand, oils affected slightly the linear growth of $F$. Oxysporum at the concentration of 2000 ppm Table(3). Oil of Syzygium aromaticum however, was the most effective inhibitor to growth of all the tested pathogens with significant or insignificant difference between its effect and the effect of the fungicide, Rizolex T 50.

Table (1): Effect of some essential oils on linear growth (cm) of $S$. rolfsii.

\begin{tabular}{|c|c|c|c|c|c|c|c|c|}
\hline \multirow[t]{3}{*}{ Essential oils } & \multicolumn{6}{|c|}{ Concentrations } & \multicolumn{2}{|c|}{ Mean } \\
\hline & \multicolumn{2}{|c|}{ 1000ppm } & \multicolumn{2}{|c|}{ 1500ppm } & \multicolumn{2}{|c|}{ 2000ppm } & & \\
\hline & L.G. & R. & L.G. & R. & L.G. & R. & L.G. & R. \\
\hline $\begin{array}{l}\text { 1- Ocimum basilicum L. 2- } \\
\text { Syzygium aromaticum L. }\end{array}$ & $\begin{array}{l}0.60 \mathrm{c} \\
0.60 \mathrm{c}\end{array}$ & $\begin{array}{l}93.33 \\
93.33\end{array}$ & $\begin{array}{l}0.60 \mathrm{c} \\
0.60 \mathrm{c}\end{array}$ & $\begin{array}{l}93.33 \\
93.33\end{array}$ & $\begin{array}{l}0.60 \mathrm{~b} \\
0.60 \mathrm{~b}\end{array}$ & $\begin{array}{l}93.33 \\
93.33\end{array}$ & $\begin{array}{l}0.60 \\
0.60\end{array}$ & $\begin{array}{l}93.33 \\
93.33\end{array}$ \\
\hline $\begin{array}{l}\text { 3- Mentha viridis } L . \\
\text { 4-Cuminum cyminum } L \text {. }\end{array}$ & $\begin{array}{l}5.05 \mathrm{~b} \\
0.63 \mathrm{c}\end{array}$ & $\begin{array}{l}43.89 \\
93.00\end{array}$ & $\begin{array}{l}1.80 \mathrm{~b} \\
0.60 \mathrm{c}\end{array}$ & $\begin{array}{l}80.00 \\
93.33\end{array}$ & $\begin{array}{l}0.60 \mathrm{~b} \\
0.60 \mathrm{~b}\end{array}$ & $\begin{array}{l}93.33 \\
93.33\end{array}$ & $\begin{array}{l}2.48 \\
0.61\end{array}$ & $\begin{array}{l}72.44 \\
93.33\end{array}$ \\
\hline $\begin{array}{l}\text { 5- Rizolex T-50. } \\
\text { 6- Control }\end{array}$ & $\begin{array}{l}0.60 \mathrm{c} \\
9.00 \mathrm{a}\end{array}$ & $\begin{array}{c}93.33 \\
0.00\end{array}$ & $\begin{array}{l}0.60 \mathrm{c} \\
9.00 \mathrm{a}\end{array}$ & $\begin{array}{l}93.33 \\
0.00 \\
\end{array}$ & $\begin{array}{l}0.60 \mathrm{~b} \\
9.00 \mathrm{a}\end{array}$ & $\begin{array}{c}93.33 \\
0.00 \\
\end{array}$ & $\begin{array}{l}0.60 \\
9.00\end{array}$ & $\begin{array}{c}93.33 \\
0.00\end{array}$ \\
\hline Mean & \multicolumn{2}{|c|}{2.75} & \multicolumn{2}{|c|}{2.20} & \multicolumn{2}{|c|}{2.00} & 2.32 & \\
\hline
\end{tabular}

Means followed by the same letter are not significantly different at $5 \%$ level by DMRT.

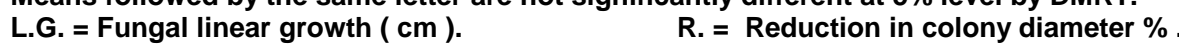


Gouda, M.l. et al.

Table (2): Effect of some essential oils on linear growth (cm) of $R$. solani.

\begin{tabular}{|c|c|c|c|c|c|c|c|c|}
\hline \multirow{3}{*}{ Essential oils } & \multicolumn{6}{|c|}{ Concentrations } & \multirow{2}{*}{\multicolumn{2}{|c|}{ Mean }} \\
\hline & \multicolumn{2}{|c|}{1000 ppm } & \multicolumn{2}{|c|}{1500 ppm } & \multicolumn{2}{|c|}{2000 ppm } & & \\
\hline & L.G. & R. & L.G. & R. & L.G. & R. & L.G. & R. \\
\hline 1-Ocimum basilicum L. 2- & $5.70 \mathrm{~b}$ & 36.67 & $0.60 \mathrm{~b}$ & 93.33 & $0.60 \mathrm{~b}$ & 93.33 & 2.30 & 74.44 \\
\hline Syzygium aromaticum L & $2.38 \mathrm{c}$ & 73.56 & $1.08 \mathrm{~b}$ & 88.00 & $0.60 \mathrm{~b}$ & 93.33 & 1.35 & 84.96 \\
\hline 3- Mentha viridis L. & $5.65 b$ & 37.22 & $0.60 \mathrm{~b}$ & 93.33 & $0.60 \mathrm{~b}$ & 93.33 & 2.28 & 74.63 \\
\hline 4-Cuminum cyminum L. & $1.25 \mathrm{~cd}$ & 86.11 & $0.60 \mathrm{~b}$ & 93.33 & $0.60 \mathrm{~b}$ & 93.33 & 0.82 & 90.82 \\
\hline 5- Rizolex T-50. & $0.60 \mathrm{~d}$ & 93.33 & $0.60 \mathrm{~b}$ & 93.33 & $0.60 \mathrm{~b}$ & 93.33 & 0.60 & 93.33 \\
\hline 6- Control & $9.00 \mathrm{a}$ & 0.00 & $9.00 \mathrm{a}$ & 0.00 & $9.00 \mathrm{a}$ & 0.00 & 9.00 & 0.00 \\
\hline \begin{tabular}{|l|} 
Mean \\
\end{tabular} & \multicolumn{2}{|c|}{4.10} & \multicolumn{2}{|c|}{2.08} & \multicolumn{2}{|c|}{2.00} & \multicolumn{2}{|c|}{2.50} \\
\hline
\end{tabular}

Means followed by the same letter are not significantly different at $5 \%$ level by DMRT.

L.G. = Fungal linear growth in (cm ) . R. = Reduction in colony diameter \% .

Table(3). Effect of essential oils on linear growth (cm) of F. oxysporum.

\begin{tabular}{|c|c|c|c|c|c|c|c|c|}
\hline \multirow{3}{*}{ Essential oils } & \multicolumn{6}{|c|}{ Concentrations } & \multirow{2}{*}{\multicolumn{2}{|c|}{ Mean }} \\
\hline & \multicolumn{2}{|c|}{1000 ppm } & \multicolumn{2}{|c|}{1500 ppm } & \multicolumn{2}{|c|}{$2000 \mathrm{ppm}$} & & \\
\hline & L.G. & R. & L.G. & R. & L.G. & R. & L.G. & R. \\
\hline 1-Ocimum basilicum L. & $5.10 \mathrm{c}$ & 41.38 & $5.00 \mathrm{~b}$ & 42.52 & $2.75 \mathrm{c}$ & 68.39 & 4.28 & 50.80 \\
\hline 2-Syzygium aromaticum L. & $2.75 \mathrm{~d}$ & 68.39 & $1.60 \mathrm{~d}$ & 81.61 & $0.60 \mathrm{e}$ & 93.33 & 1.65 & 81.11 \\
\hline 3-Mentha viridis $\mathrm{L}$. & $6.20 \mathrm{~b}$ & 28.74 & $5.20 \mathrm{~b}$ & 40.23 & $4.17 \mathrm{~b}$ & 52.07 & 5.19 & 40.35 \\
\hline 4-Cuminum cyminum $\mathrm{L}$. & $4.20 \mathrm{~cd}$ & 51.73 & $3.75 c$ & 56.90 & $1.15 \mathrm{~d}$ & 86.78 & 3.03 & 65.14 \\
\hline 5-Rizolex T- 50. & $1.40 \mathrm{e}$ & 83.90 & $1.10 \mathrm{~d}$ & 87.78 & $0.60 \mathrm{e}$ & 93.10 & 1.03 & 88.26 \\
\hline 6-Control & $8.70 \mathrm{a}$ & 0.00 & $8.70 \mathrm{a}$ & 0.00 & $8.70 \mathrm{a}$ & 0.00 & 8.70 & 0.00 \\
\hline Mean & \multicolumn{2}{|c|}{4.73} & \multicolumn{2}{|c|}{4.23} & \multicolumn{2}{|c|}{2.99} & \multicolumn{2}{|c|}{3.98} \\
\hline
\end{tabular}

Means followed by the same letter are not significantly different at $5 \%$ level by DMRT.

L.G. = Fungal linear growth in (cm ) .

R. $=\%$ reduction in colony diameter.

Table (4): Effect of essential oils on linear growth (cm) of $M$. phaseolina.

\begin{tabular}{|c|c|c|c|c|c|c|c|c|}
\hline \multirow{3}{*}{ Essential oil } & \multicolumn{6}{|c|}{ Concentrations } & \multirow{2}{*}{\multicolumn{2}{|c|}{ Mean }} \\
\hline & \multicolumn{2}{|c|}{1000 ppm } & \multicolumn{2}{|c|}{1500 ppm } & \multicolumn{2}{|c|}{2000 ppm } & & \\
\hline & L.G. & R. & L.G. & R. & L.G. & $\mathbf{R}$. & L.G. & R. \\
\hline 1- Ocimum basilicum L. & $3.53 \mathrm{c}$ & 60.78 & $0.60 \mathrm{c}$ & 3.33 & $0.60 \mathrm{~b}$ & 93.33 & 1.58 & 82.48 \\
\hline 2-Syzygium aromaticum $\mathrm{L}$. & $0.77 \mathrm{~d}$ & 91.44 & $0.60 \mathrm{c}$ & 93.33 & $0.60 \mathrm{~b}$ & 93.33 & 0.66 & 92.70 \\
\hline 3- Mentha viridis L. & $4.83 \mathrm{~b}$ & 46.33 & $0.60 \mathrm{c}$ & 93.33 & $0.60 \mathrm{~b}$ & 93.33 & 2.01 & 77.66 \\
\hline 4- Cuminum cyminum L. & $3.18 \mathrm{c}$ & 64.67 & $2.30 \mathrm{~b}$ & 74.44 & $0.60 \mathrm{~b}$ & 93.33 & 2.03 & 77.48 \\
\hline 5- Rizolex T- 50. & $0.60 \mathrm{~d}$ & 93.33 & $0.60 \mathrm{c}$ & 93.33 & $0.60 \mathrm{~b}$ & 93.33 & 0.60 & 93.33 \\
\hline 6- Control & $9.00 \mathrm{a}$ & 0.00 & $9.00 \mathrm{a}$ & 0.00 & $9.00 \mathrm{a}$ & 0.00 & 9.00 & 0.00 \\
\hline Mean & 3.65 & & 2.28 & & 2.00 & & 2.64 & \\
\hline
\end{tabular}

Means followed by the same letter are not significantly different at $5 \%$ level by DMRT.

L.G. = Fungal linear growth in (cm ) . R. = Reduction in colony diameter \% . 
Concerning the effect of the tested oils on sporulation of $F$. oxysporum, it is clear from Table (5) that all the tested oils significantly reduced number of formed spores of the fungus. However, S. aromaticum was the most effective in this respect after Rizolex T. 50.

Pot experiments : Greenhouse experiments were carried out in order to screen out the effect of essential oils on the disease incidence of sugar beet damping off and root rot caused by $S$. rolfsii and $R$. solani. Weight of roots as well as the yield components expressed as percentage of the total soluble solids (TSS) \& sucrose, purity degree of sucrose and sugar losses due to the effect of root infection was studied. S. rolfsii and $R$. solani infested potted soil was used in three replicates as mentioned under Materials and Methods. Un-infested soil and seeds treated with Rizolex T 50 served as control. Disease readings were recorded as percentage of infection after 30 days of planting for damping- off and 150 days for root rot. This experiment was done in two seasons,i.e. 2005 - 2006 and 2006 - 2007.

Table (5): Effect of some essential oils on sporulation of $F$. oxysporum.

\begin{tabular}{|c|c|c|c|c|c|c|c|c|}
\hline \multirow{3}{*}{ Essential oils } & \multicolumn{6}{|c|}{ Concentrations } & \multirow{2}{*}{\multicolumn{2}{|c|}{ Mean }} \\
\hline & \multicolumn{2}{|c|}{1000 ppm } & \multicolumn{2}{|c|}{1500 ppm } & \multicolumn{2}{|c|}{2000 ppm } & & \\
\hline & $\begin{array}{l}\text { No. } \\
\text { of sp. }\end{array}$ & R. \% & $\begin{array}{c}\text { No. } \\
\text { of sp. }\end{array}$ & R. \% & $\begin{array}{l}\text { No. } \\
\text { of sp. }\end{array}$ & R.\% & $\begin{array}{l}\text { No. } \\
\text { of sp. }\end{array}$ & R.\% \\
\hline 1- Ocimum basilicum L. & $99.00 \mathrm{~b}$ & 56.79 & $86.00 \mathrm{~b}$ & 62.28 & $63.00 \mathrm{~b}$ & 72.37 & $82.67 \mathrm{~b}$ & 63.73 \\
\hline 2- Syzygium aromaticum & $1.00 \mathrm{~cd}$ & 86.40 & $18.00 \mathrm{e}$ & 92.11 & $9.00 \mathrm{c}$ & 96.05 & $19.33 \mathrm{e}$ & 91.54 \\
\hline 3- Mentha viridis $\mathrm{L}$ & $84.00 \mathrm{~b}$ & 63.46 & $63.00 \mathrm{c}$ & 72.73 & $47.00 \mathrm{~b}$ & 79.65 & $64.67 \mathrm{c}$ & 71.62 \\
\hline 4- Cuminum cyminum L. & $42.00 \mathrm{c}$ & 81.58 & $38.00 \mathrm{~d}$ & 83.33 & $14.00 \mathrm{c}$ & 93.86 & $31.33 \mathrm{~d}$ & 86.27 \\
\hline 5- Rizolex T- 50. & $20.00 d$ & 91.23 & $11.00 \mathrm{e}$ & 95.18 & $5.00 \mathrm{c}$ & 97.81 & $12.00 \mathrm{e}$ & 94.74 \\
\hline 6- Control & $228.00 \mathrm{a}$ & 0.00 & $228.00 \mathrm{a}$ & 0.00 & $228.00 \mathrm{a}$ & 0.00 & $228.00 \mathrm{a}$ & 0.00 \\
\hline Mean & 83.0 & & 73.17 & & 60.83 & & 72.33 & \\
\hline
\end{tabular}

Means followed by the same letter are not significantly different at $5 \%$ level by DMRT.

No. of sp. = Number of spores in one square of Hemocetometer slide. $\mathbf{R}=$ Reduction in number of spores \%.

Data presented in Tables (6\&7) show that all tested essential oils have significant effect in improving the number of survived seedlings due to controlling the pre \& post - emergence damping - off. Oils of $C$. cyminum and $S$. aromaticum were highly effective in reducing damping - off and root rot as well as the severity of rot diseases of sugar beet. The rest oils were effective in decreasing the disease incidence, generally. Similar results were obtained from both seasons of experimentation.

Root fresh weight was found to be increased by decreasing the disease incidence of root rot incited by $S$. rolfsii and $R$. solani due to treating beet seeds with any of the oils tested. Tables (8\&9). It show that Cuminum cyminum and Syzygium aromaticum oils, however caused the highest degree of increasing leaf dry weight, total soluble sugars (TSS), sucrose percent in roots and sugar purity. 
Gouda, M.l. et al.

Table (6): Effect of some essential oils used for seed dressing on the incidence of sugar beet damping off, root rot and disease severity caused by $S$. rolfsii, in greenhouse, during 2005 2006 and $2006-2007$ seasons .

\begin{tabular}{|c|c|c|c|c|c|c|}
\hline \multirow[b]{2}{*}{ Treatment } & \multicolumn{2}{|c|}{ Damping-off } & \multirow[b]{2}{*}{$\begin{array}{l}\text { Surviving } \\
\text { Plants }\end{array}$} & \multicolumn{2}{|c|}{ Root rot } & \multirow[b]{2}{*}{$\begin{array}{c}\text { Healthy } \\
\text { plants }\end{array}$} \\
\hline & \begin{tabular}{|c|} 
pre- \\
emergence \\
$\%$
\end{tabular} & \begin{tabular}{|c|} 
Post- \\
emergence \\
$\%$
\end{tabular} & & $\begin{array}{c}\text { Disease } \\
\text { incidence } \\
\%\end{array}$ & $\begin{array}{c}\text { Disease } \\
\text { Severity } \\
\%\end{array}$ & \\
\hline \multicolumn{7}{|c|}{$2005-2006$ season } \\
\hline $\begin{array}{l}\text { 1- Ocimum basilicum L. } \\
\text { 2-Syzygium aromaticum. } \\
\text { 3- Mentha viridis L } \\
\text { 4- Cuminum cyminum L } \\
\text { 5- Rizolex T-50. } \\
\text { 6- Control : Infested } \\
\text { 7- Control : Uninfested }\end{array}$ & $\begin{array}{c}37.03 \mathrm{c} \\
0.00 \mathrm{a} \\
25.92 \mathrm{~b} \\
0.00 \mathrm{a} \\
0.00 \mathrm{a} \\
96.30 \mathrm{~d} \\
0.00 \mathrm{a}\end{array}$ & \begin{tabular}{|c|}
$25.94 \mathrm{~d}$ \\
$33.34 \mathrm{e}$ \\
$11.11 \mathrm{c}$ \\
$25.92 \mathrm{~d}$ \\
$7.41 \mathrm{bc}$ \\
$0.00 \mathrm{a}$ \\
$0.00 \mathrm{a}$ \\
\end{tabular} & \begin{tabular}{|c|}
$37.03 \mathrm{~b}$ \\
$66.66 \mathrm{~d}$ \\
$62.97 \mathrm{c}$ \\
$74.08 \mathrm{e}$ \\
$92.59 \mathrm{f}$ \\
$3.70 \mathrm{a}$ \\
$100.00 \mathrm{~g}$
\end{tabular} & $\begin{array}{c}44.44 \mathrm{c} \\
11.11 \mathrm{~b} \\
44.44 \mathrm{c} \\
0.00 \mathrm{a} \\
0.00 \mathrm{a} \\
100.00 \mathrm{~d} \\
0.00 \mathrm{a}\end{array}$ & $\begin{array}{l}4.67 \mathrm{~b} \\
0.67 \mathrm{ab} \\
5.17 \mathrm{c} \\
0.00 \mathrm{a} \\
0.00 \mathrm{a} \\
7.67 \mathrm{~d} \\
0.00 \mathrm{a}\end{array}$ & $\begin{array}{c}55.56 \mathrm{~b} \\
88.89 \mathrm{~d} \\
55.56 \mathrm{~b} \\
100.00 \mathrm{c} \\
100.00 \mathrm{c} \\
0.00 \mathrm{a} \\
100.00 \mathrm{c}\end{array}$ \\
\hline \multicolumn{7}{|c|}{$2006-2007$ season } \\
\hline $\begin{array}{l}\text { 1- Ocimum basilicum L. } \\
\text { 2- Syzygium aromaticum. } \\
\text { 3- Mentha viridis L } \\
\text { 4- Cuminum cyminum L } \\
\text { 5- Rizolex T-50. } \\
\text { 6- Control : Infested } \\
\text { 7- Control : Uninfested }\end{array}$ & $\begin{array}{r}45.15 \mathrm{c} \\
0.00 \mathrm{a} \\
48.15 \mathrm{~d} \\
6.41 \mathrm{~b} \\
0.00 \mathrm{a} \\
77.77 \mathrm{e} \\
0.00 \mathrm{a}\end{array}$ & \begin{tabular}{|c|}
$17.82 \mathrm{c}$ \\
$30.33 \mathrm{~d}$ \\
$18.52 \mathrm{~cd}$ \\
$33.33 \mathrm{e}$ \\
$14.82 \mathrm{~b}$ \\
$14.82 \mathrm{~b}$ \\
$0.00 \mathrm{a}$
\end{tabular} & \begin{tabular}{|c|}
$37.03 \mathrm{c}$ \\
$69.67 \mathrm{e}$ \\
$33.33 \mathrm{bc}$ \\
$60.26 \mathrm{~d}$ \\
$85.18 \mathrm{f}$ \\
$7.41 \mathrm{a}$ \\
$100.00 \mathrm{~h}$
\end{tabular} & $\begin{array}{c}44.44 \mathrm{~d} \\
11.11 \mathrm{~b} \\
33.33 \mathrm{c} \\
0.00 \mathrm{a} \\
0.00 \mathrm{a} \\
88.89 \mathrm{e} \\
0.00 \mathrm{a}\end{array}$ & $\begin{array}{l}4.83 \mathrm{~b} \\
0.67 \mathrm{ab} \\
6.33 \mathrm{c} \\
0.00 \mathrm{a} \\
0.00 \mathrm{a} \\
8.00 \mathrm{~d} \\
0.00 \mathrm{a}\end{array}$ & $\begin{array}{c}55.56 \mathrm{~b} \\
88.89 \mathrm{~d} \\
66.67 \mathrm{c} \\
100.00 \mathrm{e} \\
100.00 \mathrm{e} \\
11.11 \mathrm{a} \\
100.00 \mathrm{e}\end{array}$ \\
\hline
\end{tabular}

Means followed by the same letter are not significantly different at $5 \%$ level by DMRT.

Table (7): Effect of some essential oils used for seed dressing on the incidenc of sugar beet damping-off, root rot and disease severity caused by $R$. solani, in greenhouse, during 2005 2006 and 2006 - 2007 seasons .

\begin{tabular}{|c|c|c|c|c|c|c|}
\hline \multirow[b]{2}{*}{ Treatment } & \multicolumn{2}{|c|}{ Damping-off } & \multirow[b]{2}{*}{$\begin{array}{l}\text { Survivin } \\
\text { g plants }\end{array}$} & \multicolumn{2}{|c|}{ Root rot } & \multirow[b]{2}{*}{$\begin{array}{c}\text { Healthy } \\
\text { plants }\end{array}$} \\
\hline & $\begin{array}{c}\text { pre- } \\
\text { emergence } \\
\%\end{array}$ & $\begin{array}{c}\text { Post- } \\
\text { emergence } \\
\%\end{array}$ & & $\begin{array}{c}\text { Disease } \\
\text { incidence } \\
\%\end{array}$ & $\begin{array}{l}\text { Disease } \\
\text { severity }\end{array}$ & \\
\hline \multicolumn{7}{|c|}{2005 - 2006 season } \\
\hline 1- Ocimum basilicum L. & $51.85 \mathrm{~d}$ & $18.52 \mathrm{~d}$ & $29.63 \mathrm{~b}$ & $22.22 \mathrm{c}$ & $1.33 \mathrm{~b}$ & $77.78 \mathrm{c}$ \\
\hline 2- Syzygium aromaticum. & $37.04 \mathrm{~b}$ & $22.22 \mathrm{de}$ & $40.74 \mathrm{c}$ & $11.11 \mathrm{~b}$ & $0.33 \mathrm{a}$ & $88.89 d$ \\
\hline 3- Mentha viridis $L$ & $44.45 \mathrm{c}$ & $25.92 \mathrm{e}$ & $29.63 \mathrm{~b}$ & $33.33 \mathrm{~d}$ & $1.67 \mathrm{bc}$ & $66.67 \mathrm{~b}$ \\
\hline 4- Cuminum cyminum L. & $37.04 \mathrm{~b}$ & $11.11 \mathrm{c}$ & $51.85 d$ & $11.11 \mathrm{~b}$ & $0.33 \mathrm{a}$ & $88.89 \mathrm{~d}$ \\
\hline 5- Rizolex T-50. & $0.00 \mathrm{a}$ & $3.67 \mathrm{~b}$ & $96.33 \mathrm{e}$ & $0.00 \mathrm{a}$ & $0.00 \mathrm{a}$ & $100.00 \mathrm{e}$ \\
\hline 6- Control : Infested & $96.30 \mathrm{e}$ & $0.00 \mathrm{a}$ & $3.70 \mathrm{a}$ & $100.00 \mathrm{e}$ & $7.17 \mathrm{c}$ & $0.00 \mathrm{a}$ \\
\hline 7- Control : Uninfested & $0.00 \mathrm{a}$ & $0.00 \mathrm{a}$ & $100.00 \mathrm{f}$ & $0.00 \mathrm{a}$ & $0.00 \mathrm{a}$ & $100.00 \mathrm{e}$ \\
\hline \multicolumn{7}{|c|}{2006 - 2007season } \\
\hline 1- Ocimum basilicum L. & $44.44 \mathrm{c}$ & $18.52 \mathrm{f}$ & $37.04 \mathrm{~b}$ & $33.33 \mathrm{c}$ & $2.33 \mathrm{c}$ & $66.67 c$ \\
\hline 2- Syzygium aromaticum. & $33.33 \mathrm{~b}$ & $22.22 \mathrm{~g}$ & $44.45 \mathrm{c}$ & $16.00 \mathrm{~b}$ & $1.33 \mathrm{~b}$ & $84.00 \mathrm{~d}$ \\
\hline 3- Mentha viridis $\mathrm{L}$ & $33.33 \mathrm{~b}$ & $11.11 \mathrm{~d}$ & $55.56 \mathrm{~d}$ & $44.44 d$ & $2.67 \mathrm{~cd}$ & $55.56 \mathrm{~b}$ \\
\hline 4- Cuminum cyminum $\mathrm{L}$. & $33.33 \mathrm{~b}$ & $14.81 \mathrm{e}$ & $51.86 \mathrm{e}$ & $0.00 \mathrm{a}$ & $0.00 \mathrm{a}$ & $100.00 \mathrm{e}$ \\
\hline 5- Rizolex T-50. & $0.00 \mathrm{a}$ & $7.41 \mathrm{c}$ & $92.59 \mathrm{f}$ & $0.00 \mathrm{a}$ & $0.00 \mathrm{a}$ & $100.00 \mathrm{e}$ \\
\hline 6- Control : Infested & $85.19 d$ & $3.67 \mathrm{~b}$ & $11.14 \mathrm{a}$ & $88.89 \mathrm{e}$ & $6.67 \mathrm{~d}$ & $11.11 \mathrm{a}$ \\
\hline 7- Control : Uninfested & $0.00 \mathrm{a}$ & $0.00 \mathrm{a}$ & $100.00 \mathrm{~g}$ & $0.00 \mathrm{a}$ & $0.00 \mathrm{a}$ & $100.00 \mathrm{e}$ \\
\hline
\end{tabular}

Mean followed by the same letter are not significantly different at the $5 \%$ level by DMRT. 
J. Agric. Sci. Mansoura Univ., 34 (4), April, 2009

T8

3831 
Gouda, M.I. et al.

T9

3832 
Field experiment : Different essential oils were studied for their effect on root rot of sugar beet under natural infection at the Farm of Sakha in 20052006 and 2006-2007 seasons.

Parameters of plant growth were studied in the same two growing seasons and data are shown in Table (10). Results indicate that all oils improved plant growth expressed as plant height, leaf area, and leaf dry weight. Rizolex T 50 enhancing the plant growth comparable to the other matters. This is correct in both seasons of experimentation.

Table (10): Effect of some essential oils on parameters of plant growth sugar beet, under field conditions, Sakha, 2005 - 2006 and 2006 - 2007 seasons.

\begin{tabular}{|c|c|c|c|c|c|c|}
\hline \multirow[b]{2}{*}{ Treatment } & \multicolumn{3}{|c|}{2005 - 2006 season } & \multicolumn{3}{|c|}{2006 - 2007 season } \\
\hline & $\begin{array}{l}\text { Plant } \\
\text { height } \\
\text { (cm) }\end{array}$ & $\begin{array}{c}\text { Leaf } \\
\text { area } \\
\left(\mathrm{cm}^{2}\right)\end{array}$ & $\begin{array}{c}\text { Dry } \\
\text { weight } \\
\text { (g) }\end{array}$ & $\begin{array}{l}\text { Plant } \\
\text { height } \\
\text { (cm) }\end{array}$ & $\begin{array}{c}\text { Leaf } \\
\text { area } \\
\left(\mathrm{cm}^{2}\right)\end{array}$ & $\begin{array}{c}\text { Dry } \\
\text { weight } \\
\text { (g) }\end{array}$ \\
\hline 1- Ocimum basilicum L. & $37.67 d$ & $818.63 \mathrm{~d}$ & $11.07 \mathrm{~b}$ & $36.67 \mathrm{~d}$ & $977.71 \mathrm{~cd}$ & $10.03 \mathrm{~cd}$ \\
\hline $\begin{array}{l}\text { 2-Syzygium aromaticum } \mathrm{L} \text {. } \\
\text { 3- Mentha viridis } \mathrm{L} \\
\text { 4- Cuminum cyminum L. } \\
\text { 5- Rizolex T-50. }\end{array}$ & $\begin{array}{c}51.33 b \\
42.00 c \\
39.67 c d \\
64.67 a\end{array}$ & $\begin{array}{c}1303.19 \mathrm{a} \\
977.09 \mathrm{c} \\
1081.68 \mathrm{bc} \\
1128.11 \mathrm{~b}\end{array}$ & $\begin{array}{c}12.63 \mathrm{a} \\
10.73 \mathrm{c} \\
9.37 \mathrm{~d} \\
12.07 \mathrm{ab}\end{array}$ & $\begin{array}{c}51.00 \mathrm{~b} \\
45.00 \mathrm{c} \\
48.00 \mathrm{bc} \\
54.67 \mathrm{a}\end{array}$ & $\begin{array}{c}1108.58 \mathrm{a} \\
986.86 \mathrm{c} \\
1061.97 \mathrm{~b} \\
1098.30 \mathrm{ab}\end{array}$ & $\begin{array}{l}11.63 \mathrm{a} \\
10.13 \mathrm{c} \\
8.77 \mathrm{~d} \\
11.23 \mathrm{~b}\end{array}$ \\
\hline 6- Control & $25.00 \mathrm{e}$ & $655.13 \mathrm{e}$ & $7.07 \mathrm{e}$ & $22.33 \mathrm{e}$ & $554.05 \mathrm{e}$ & $6.30 \mathrm{e}$ \\
\hline
\end{tabular}

Mean followed by the same letter are not significantly different at the $5 \%$ level by DMRT.

Data presented in Table (11) reveal that oils of $S$. aromaticum \& $C$. cyminum were superior than the other materials in reducing the root rot of sugar beet as well as the disease severity in both seasons of experimentation. The yield per plot was found also to be increased due to treatment with these materials. Rizolex T 50 caused the least level of infection and disease severity if compared with the other treatments.

Table (11): Effect of some essential oils on root rot diseases under field conditions,Sakha, 2005 - 2006 and 2006 - 2007 seasons.

\begin{tabular}{|c|c|c|c|c|c|c|}
\hline \multirow[b]{2}{*}{ Treatments } & \multicolumn{3}{|c|}{2005 - 2006 season } & \multicolumn{3}{|c|}{2006 - 2007 season } \\
\hline & $\begin{array}{c}\text { Disease } \\
\text { incidence } \\
\%\end{array}$ & $\begin{array}{c}\text { Disease } \\
\text { severity } \\
\%\end{array}$ & $\begin{array}{l}\text { Root rot } \\
\text { Yield/plot } \\
\text { (kg) }\end{array}$ & $\begin{array}{c}\text { Disease } \\
\text { incidence } \\
\%\end{array}$ & $\begin{array}{c}\text { Disease } \\
\text { severity } \\
\%\end{array}$ & $\begin{array}{l}\text { Root rot } \\
\text { Yield/plot } \\
\text { (kg) }\end{array}$ \\
\hline 1- Ocimum basilicum L. & $2.33 \mathrm{c}$ & $1.33 \mathrm{c}$ & $52.00 \mathrm{~b}$ & $1.24 \mathrm{c}$ & $0.67 \mathrm{bc}$ & $54.00 \mathrm{~b}$ \\
\hline 2-Syzygium aromaticum & $0.54 a b$ & $0.33 \mathrm{a}$ & $54.00 \mathrm{~b}$ & $0.66 \mathrm{a}$ & $0.33 \mathrm{a}$ & $56.00 \mathrm{~b}$ \\
\hline 3- Mentha viridis $L$. & $1.84 \mathrm{~b}$ & $0.83 \mathrm{~b}$ & $42.00 \mathrm{c}$ & $1.31 \mathrm{c}$ & $0.83 c$ & $46.00 \mathrm{c}$ \\
\hline 4- Cuminum cyminum L. & $0.65 a$ & $0.33 \mathrm{a}$ & $52.00 \mathrm{~b}$ & $0.57 \mathrm{~b}$ & $0.33 \mathrm{a}$ & $46.00 \mathrm{c}$ \\
\hline 5- Rizolex T-50* & $0.63 \mathrm{a}$ & $0.20 \mathrm{a}$ & $64.00 \mathrm{a}$ & $0.65 \mathrm{a}$ & $0.50 \mathrm{~b}$ & $64.02 \mathrm{a}$ \\
\hline 6- Control & $8.05 d$ & $3.67 \mathrm{~d}$ & $36.00 \mathrm{~d}$ & $9.32 \mathrm{~d}$ & $3.67 \mathrm{~d}$ & $26.00 \mathrm{~d}$ \\
\hline
\end{tabular}

Means followde by the same letter are not significantly different at the $5 \%$ level by DMRT.

\section{DISCUSSION}

Trials were conducted to study the possibility of controlling sugar beet damping off and root rots caused by some soil borne fungi. The essential oils tested inhibited growth of the fungal mycelium in Vitro. Also the tested oils could successfully reduce damping - off and root rots of sugar beet in the greenhouse and field. Yield per plot was also significantly increased due to 
these applications. Syzygium aromaticum. L, Cuminum cyminum. L were the superior among all materials in suppressing damping off and root rots in greenhouse and field. Its positive effect against sugar beet root diseases reflects, in turn on the root yield, whereas, it improved the yield potentiality comparable to the untreated control.

Parameters of plant growth were enhanced due to these treatments. Increasing in total soluble sugars (TSS) and sugar purity in roots due to these applications were observed. This result causes, in turn an improve to the sugar quality within the roots. These results are consistent with those obtained by other investigators who found an antimicrobial activity of some oils against many of pathogens in vitro (Madhukor and Reddy, 1989 ; Farag et al.,1989; Deans et al., 1992; Mc Cutcheon et al., 1994 and Navarro et al.,1996). Some essential oils have an allelopathic effect on some diseases on other plant hosts as previous investigators have reported (Jain, et al.,1992; Paran et al.,1996; El-Shoraky 1998 and Gouda, 2001). ElKazzaz et al., 2003 studied the effect of essential oil, Mint, Clove, Cumin and Basil on Sclerotium rolfsii Sacc., the causal pathogen of sugar beet damping- off and root rot diseae. they found that all of these oils inhibited the linear growth and sporulation at the concentration of $1000 \mathrm{ppm}$. Similer results were found by Taha, 2004 on Sclerotium sclerotiourm the causal pathogen of white rot disease of many vegetable crops.

Based on the obtained rusltus, it is recommended to use $S$. aromaticum \& C. cyminum in controlling the major pathogens of the root rots of sugar beet. This oils offer an excellent sourse of biologically active natural product through its allelopathic effect . Allelopathy, as defined by Rice (1984) is any direct or indirect benficial or harmful effect of one organism (including plant or microorganism) on other through release of chemicals into the environment. It is grown mainly in the Nile region and in some regional and global countries. Seeds of this medicinal plant are available and cheap in local market. Active constituents that have the allelopathic effect of seeds according to Batanouny, et al., (1999) . Other oils used in this study) may be attributed to the known and unknown chemical compounds having synergistic effect on the pathogen. Besides, they may affect the populations of soil microflora around the host roots which may cause, in turn a rise of antagonistic and biological agents. Therefore, the authors highly recommends, in the time being to soak seed of sugar beet with oils for $8 \mathrm{~h}$ before planting for satisfactory control of such disease in the field. It is worth mentioning that using other means of disease control rather than fungicides is strongly encouraged by the government to decrease environmental pollution caused by fungicides.

\section{REFERENCES}

Aly, A. A.; E.M. Hussein ; M.A. Mostafa and A.I. Ismail (1996). Distribution, identification and pathogenicity of Fusarium spp. isolated from some Egyptian cottons. Menofiya Journal of Agricultural Res., 21 (4) : 819 835. 
A.O.A.C., Association of official analytical chemists (1990). Official methods analysis of the association of official analytical chemists. Washinton 25. D. C., USA

Batanouny, K.H.; E. Aboutabl ; M. Shabana and F. Soliman (1999). Wild Medicinal Plants in Egypt. Academy of Scientific Research and Technology, Egypt, $207 \mathrm{pp}$.

Deans, S.G. ; K.P. Sovboda ; M. Gundidza and E.Y. Brechany. (1992). Essential oil profiles of several temperate and tropical aromatic plants: their antimicrobial and antioxidant activities. International symposium on medicinal and aromatic plants, Budapest, Hungary, $4-6$ sep, Acta -Horticulturae, No. 306, 229 -232.

Eah, A. M. H. E. (2000). Studies on some sugar beet root diseases in Egypt. $\mathrm{Ph}$.D. Thesis, Faculty of Agric. Zagazig Univ., 287pp.

El-Abyad, M.S. ; A.M. Abu-Taleb and M.S. Khalil (1992). Impact of salinity stress on soil borne fungi of sugar beet III. Plant cell wall-degrading enzymes by Rhizoctonia solani Kuhn and Sclerotium rolfsii Sacc. In vivo and In vitro. Plant soil, 143 (1) : 75 - 83.

El-Kazzaz, M.K. ; M.A. Hassan ; M.M. Badr and K.E. Ghoniem (1999). Studies on sugar beet root diseases in Northern Nile Delta. J. Agric. Res., Tanta Univ., 25 (2) : 122 -131.

El-Kazzaz, M.K.; E.M. El-Assiuty ; M.M. Badr ; H. El-Zahaby and M.I. Gouda (2003). Effect of some plant extracts and essential oils on controlling sugar beet root rot disease caused by Sclerotium rolfsii Sacc. In proceeding of the $10^{\text {th }}$ Congress of the Egyptian Phytopathological Society, 9 -10 Dec. 2003, Giza, Egypt.

El-Kholi, M.M.A. (2000). Sugar beet diseases in Egypt. The Ninth Conference of Phytopathology. The Egypt. Phytopathol. Soc., Giza, Egypt, May 2000.

El-Shoraky, Fathia, S.A. (1998). Using extracts and oils of some plants in controlling plant diseases. Ph.D. Thesis, Faculty of Agric. Kafr ElSheikh, Tanta University, $187 \mathrm{pp}$.

Farag, R.S. ; Z.Y. Daw ;F.M. Hewedi and G.S.A. El-Baroty. (1989). Antimicrobial activity of some spice essential oils. Journal of Protection, 52 (9) : 665 - 667.

Gouda. M.I. (2001). Studies on some causals of sugar beet root rots. Ph.D. Thesis, Faculty of Agric., Tanta Univ. Egypt. 146 pp.

Jain, S. C. ; M. Purohit and R. Jain. (1992) Pharmacological evaluation of Cuminum cyminum. Fitoterapia., 63 (4) : 291-294.

Madhukor, J. and S.M. Reddy . (1989). Efficacy of certain oils in the control of fruit rot of guava. Indian Journal of Mycology and Plant Pathology, 19 (1) : 131-132.

Mandeel, Q. and R. Baker (1991). Mechanisms involved in biological control of Fusarium wilt of cucumber with strains of nonpathogenic Fusarium oxysporum. Phytopathology. 81 (4) : 462 - 469.

Mc Cutcheon, A.R. ; S.M. Ellis ; R.E.W. ; Hancock and G.H.N. Towers (1994). Antifungal screening of medicinal plant of British Columbian native peoples. Journal of Ethno pharmacology, 44 (3) : 157-169 
Gouda, M.l. et al.

Mc Ginnis, R.A. (1982). Beet sugar technology. 3rd edn. Beet sugar development Foundation for Collins, $855 \mathrm{pp}$.

Navarro,V.; M.L.Villarreal; G.Rojas and X. Lozoya (1996). Antimicrobial evaluation of some plants used in Mexicen traditonal medicine for the treatment infectious diseases. Journal of Ethno pharmacology, 53 (3) :143 -147.

Paran, B.; R.K. Sharma; R.S. Singh and A.C. Ghosh (1996). Fungicidal activity of some naturally occurring essential oils against Fusarium moniliforme. J. Essential Oil. Res., 8 (4) : 411- 412.(c.f.Rev.PI. path.,76 (2)125 - 126).

Rice, E.L. (1984). Allelopathy. 2nd edn. Academic Press, New York. 422 pp. Sharma, B. S. and V. N. Pathak ( 1994 ). Yield and sucrose loss in sugar to root rot. Indian phytopathology, 47 ( 4 ) : $408-411$.

Taha. Naglaa, A. A. (2004). Integrated control of Sclerotinia sclerotiorum the soil-borne pathogen which affecting certain vegetable crops M. Sc. Thesis, Fac. Of Agric ., Tanta Univ., Egypt. 85 pp.

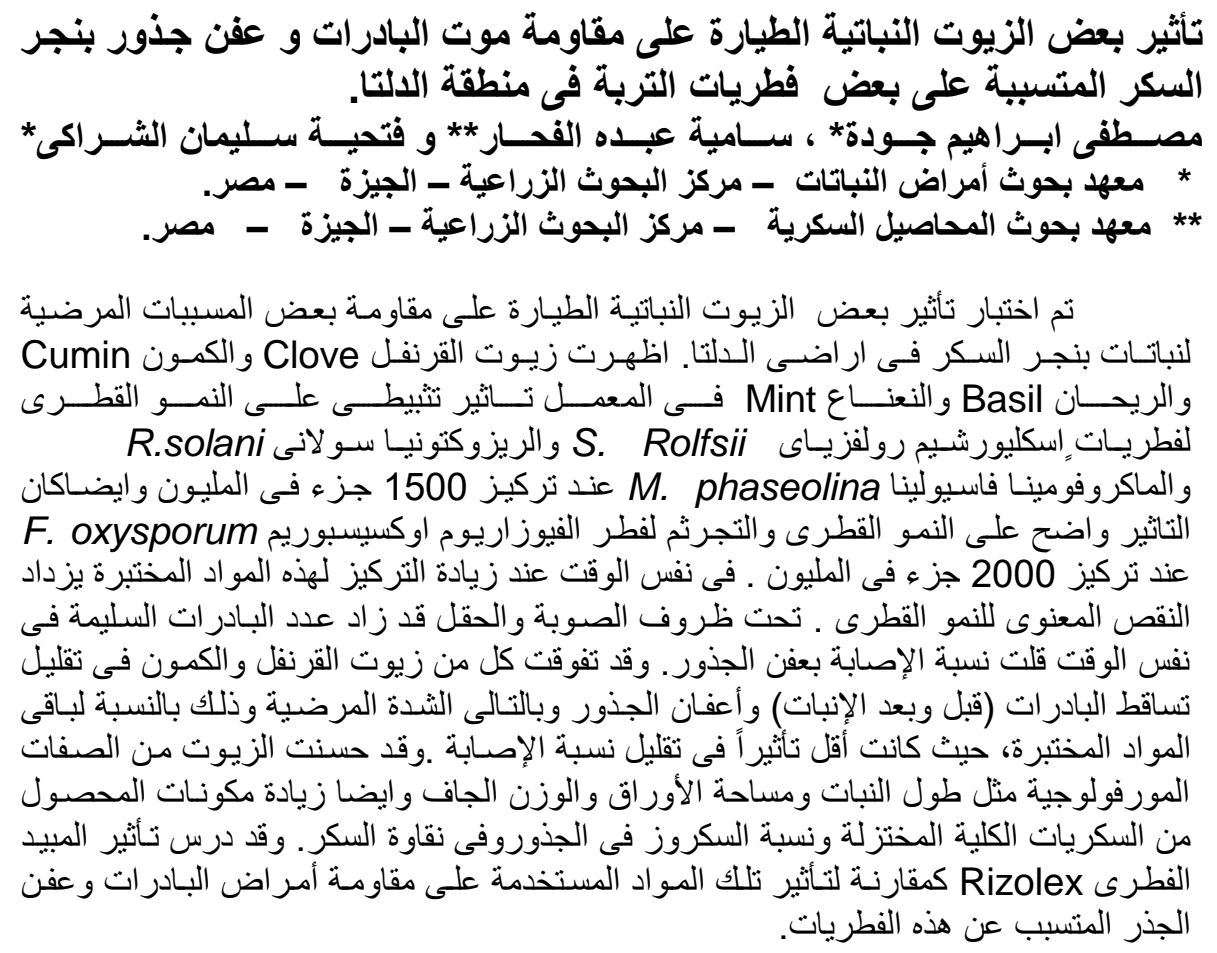


Table (8): Effect of some essential oils on disease incidence, disease severity, root weight / plant, percentage of total soluble solids (TSS), percentage of sucrose, purity and losses (\%) in yield and sucrose of sugar beet root rot under artificial infestation with S. rolfsii, in greenhouse during 2005 - 2006 and 2006 2007 seasons .

\begin{tabular}{|c|c|c|c|c|c|c|c|c|c|c|c|c|}
\hline \multirow[t]{2}{*}{ Treatment } & \multirow{2}{*}{$\begin{array}{c}\text { Disease } \\
\text { incidence }\end{array}$} & \multirow{2}{*}{$\begin{array}{l}\text { Disease } \\
\text { severity }\end{array}$} & \multicolumn{2}{|c|}{$\begin{array}{c}\text { Root } \\
\text { weight/plant }\end{array}$} & \multicolumn{2}{|c|}{ TSS } & \multicolumn{2}{|c|}{$\begin{array}{c}\text { Sucrose } \\
\%\end{array}$} & \multicolumn{2}{|c|}{$\underset{\%}{\text { Purity }}$} & \multicolumn{2}{|c|}{$\begin{array}{l}\text { Losses } \\
\%\end{array}$} \\
\hline & & & Infected & Helthy & Infected & Helthy & Infected & Helthy & Infected & Helthy & Yield & Sucrose \\
\hline \multicolumn{13}{|c|}{$2005-2006$} \\
\hline 1-Ocimum basilicum & $44.44 \mathrm{c}$ & $4.67 \mathrm{~b}$ & $0.597 \mathrm{e}$ & $1.095 \mathrm{~cd}$ & $12.27 \mathrm{e}$ & $18.33 \mathrm{c}$ & $9.47 \mathrm{e}$ & $14.80 \mathrm{~b}$ & 77.18 & 80.74 & 45.47 & 36.01 \\
\hline 2- Syzygium aromaticum. & $11.11 \mathrm{~b}$ & $0.67 \mathrm{a}$ & $0.929 \mathrm{c}$ & $1.115 \mathrm{c}$ & $16.27 \mathrm{de}$ & $17.87 d$ & $13.00 \mathrm{~b}$ & $15.60 \mathrm{a}$ & 79.90 & 87.30 & 16.68 & 16.10 \\
\hline 3- Mentha viridis $\mathrm{L}$ & $54.44 \mathrm{~d}$ & $5.17 \mathrm{c}$ & $0.487 f$ & $0.809 \mathrm{e}$ & $12.07 \mathrm{e}$ & $18.07 \mathrm{c}$ & $10.73 d$ & $14.40 \mathrm{~d}$ & 59.30 & 80.20 & 39.80 & 25.48 \\
\hline 4- Cuminum cyminum L. & $0.00 \mathrm{a}$ & $.00 \mathrm{a}$ & $0.890 \mathrm{~d}$ & $0.927 d$ & $17.73 \mathrm{c}$ & $19.27 b$ & $11.40 \mathrm{c}$ & $14.20 \mathrm{de}$ & 64.30 & 73.69 & 3.99 & 19.70 \\
\hline 5- Rizolex T-50. & $0.00 \mathrm{a}$ & $0.00 \mathrm{a}$ & $1.380 \mathrm{a}$ & $1.412 \mathrm{a}$ & $20.00 \mathrm{a}$ & $20.72 \mathrm{a}$ & $15.00 \mathrm{a}$ & $15.60 \mathrm{a}$ & 75.00 & 75.25 & 2.27 & 3.61 \\
\hline 6- Control : Infested & $100.00 \mathrm{e}$ & $7.67 \mathrm{~d}$ & $0.237 \mathrm{~g}$ & $1.355 \mathrm{~b}$ & $6.40 \mathrm{~g}$ & $19.40 \mathrm{~b}$ & $3.53 f$ & $15.40 \mathrm{a}$ & 55.15 & 79.38 & 82.50 & 77.00 \\
\hline 7-Control: Uninfested & $0.00 \mathrm{a}$ & $0.00 \mathrm{a}$ & $1.355 \mathrm{~b}$ & $1.355 \mathrm{~b}$ & $19.60 \mathrm{~b}$ & $19.60 \mathrm{~b}$ & $15.40 \mathrm{a}$ & $15.40 \mathrm{a}$ & 78.57 & 78.57 & 0.00 & 0.00 \\
\hline \multicolumn{13}{|c|}{$2006-2007$} \\
\hline 1- Ocimum basilicum & $44.44 d$ & $4.83 \mathrm{~b}$ & $0.440 \mathrm{e}$ & $1.102 \mathrm{c}$ & $10.67 \mathrm{~g}$ & 19.00de & $8.33 \mathrm{e}$ & $16.20 \mathrm{c}$ & 78.06 & 88.42 & 60.07 & 50.42 \\
\hline 2-Syzygium aromaticum. & $11.11 \mathrm{~b}$ & $0.67 \mathrm{ab}$ & $0.830 \mathrm{c}$ & $1.071 \mathrm{c}$ & $15.20 \mathrm{~d}$ & $19.33 \mathrm{~cd}$ & $13.20 \mathrm{c}$ & $18.67 \mathrm{a}$ & 86.84 & 96.58 & 22.50 & 29.29 \\
\hline 3- Mentha viridis $\mathrm{L}$ & $33.33 \mathrm{c}$ & $6.33 \mathrm{c}$ & $0.570 \mathrm{~d}$ & $0.911 d$ & $10.73 \mathrm{~g}$ & $18.07 f$ & $6.73 f$ & $16.03 d$ & 62.72 & 88.71 & 37.43 & 58.01 \\
\hline 4-Cuminum cyminum $\mathrm{L}$. & $0.00 \mathrm{a}$ & $0.00 \mathrm{a}$ & $0.820 \mathrm{c}$ & $0.939 \mathrm{~d}$ & $16.67 \mathrm{c}$ & $17.75 f$ & $12.60 \mathrm{~d}$ & $13.40 \mathrm{e}$ & 75.58 & 75.57 & 0.67 & 5.97 \\
\hline 5- Rizolex T-50. & $0.00 \mathrm{a}$ & $0.00 \mathrm{a}$ & $1.475 \mathrm{a}$ & $1.484 \mathrm{a}$ & $20.60 \mathrm{a}$ & $20.17 \mathrm{a}$ & $17.47 \mathrm{a}$ & $17.93 \mathrm{~b}$ & 84.81 & 85.91 & 0.61 & 2.57 \\
\hline 6- Control : Infested & $8889 \mathrm{e}$ & $8.00 \mathrm{~d}$ & $0.190 \mathrm{f}$ & $1.240 \mathrm{~b}$ & $4.47 \mathrm{j}$ & $19.27 \mathrm{de}$ & $2.40 \mathrm{~g}$ & $16.60 \mathrm{c}$ & 53.69 & 86.14 & 84.67 & 85.50 \\
\hline 7- Control: Uninfested & $0.00 \mathrm{a}$ & $0.00 \mathrm{a}$ & $1.240 \mathrm{~b}$ & $1.240 \mathrm{~b}$ & $19.27 \mathrm{~b}$ & $19.27 \mathrm{de}$ & $16.60 \mathrm{~b}$ & $16.60 \mathrm{c}$ & 26.14 & 86.14 & 0.00 & 0.00 \\
\hline
\end{tabular}

Mean followed by the same letter are not significantly different at the $5 \%$ level by DMRT. 
Table (9): Effect of some essential oils on disease incidence, disease severity, root weight / plant, percentage of total soluble solids (TSS), percentage of sucrose, purity and losses (\%) in yield and sucrose of sugar beet root rot under artificial infestation with R. solani, in greenhouse during 2005 - 2006 and 2006 2007 seasons.

\begin{tabular}{|c|c|c|c|c|c|c|c|c|c|c|c|c|}
\hline \multirow[t]{2}{*}{ Treatment } & \multirow{2}{*}{$\begin{array}{c}\text { Disease } \\
\text { incidence }\end{array}$} & \multirow{2}{*}{$\begin{array}{l}\text { Disease } \\
\text { severity }\end{array}$} & \multicolumn{2}{|c|}{$\begin{array}{c}\text { Root } \\
\text { weight/plant }\end{array}$} & \multicolumn{2}{|c|}{ TSS } & \multicolumn{2}{|c|}{$\begin{array}{c}\text { Sucrose } \\
\%\end{array}$} & \multicolumn{2}{|c|}{$\begin{array}{c}\text { Purity } \\
\%\end{array}$} & \multicolumn{2}{|c|}{$\begin{array}{c}\text { Losses } \\
\%\end{array}$} \\
\hline & & & Infected & Helthy & Infected & Helthy & Infected & Helthy & Infected & Helthy & Yield & Sucrose \\
\hline \multicolumn{13}{|c|}{$2005-2006$} \\
\hline 1- Ocimum basilicum & $22.22 \mathrm{c}$ & $1.33 \mathrm{bc}$ & $1.018 \mathrm{~d}$ & $1.318 \mathrm{c}$ & 16.20 & $18.00 \mathrm{bc}$ & $11.67 \mathrm{c}$ & $16.03 a$ & 72.03 & 89.06 & 22.76 & 27.19 \\
\hline 2- Syzygium aromaticum $\mathrm{L}$. & $11.11 \mathrm{~b}$ & $0.33 \mathrm{a}$ & $1.237 \mathrm{c}$ & $1.420 \mathrm{~b}$ & $17.20 \mathrm{~b}$ & $17.67 \mathrm{~cd}$ & $12.87 \mathrm{~b}$ & $15.00 \mathrm{bc}$ & 74.82 & 84.89 & 12.88 & 14.20 \\
\hline 3- Mentha viridis $\mathrm{L}$. & $33.33 d$ & $1.67 \mathrm{~cd}$ & $1.133 \mathrm{c}$ & $1.528 \mathrm{a}$ & $15.20 \mathrm{e}$ & $18.40 \mathrm{a}$ & $12.67 \mathrm{~b}$ & $13.33 f$ & 72.45 & 83.35 & 25.85 & 4.95 \\
\hline 4- Cuminum cyminum $\mathrm{L}$ & $11.11 \mathrm{~b}$ & $0.33 \mathrm{a}$ & $1.123 \mathrm{~cd}$ & 1.278 & $15.60 f$ & $18.20 \mathrm{~b}$ & 12.40 & $14.07 \mathrm{~d}$ & 79.48 & 77.31 & 12.12 & 27.19 \\
\hline 5- Rizolex T-50. & $0.00 \mathrm{a}$ & $0.00 \mathrm{a}$ & $1.502 \mathrm{a}$ & $1.518 \mathrm{a}$ & $17.80 \mathrm{a}$ & $18.27 \mathrm{ab}$ & $14.60 \mathrm{a}$ & 14.73 & 79.91 & 80,62 & 0.10 & 0.88 \\
\hline 6- Control : Infested & 82.22 & $7.17 \mathrm{e}$ & 0.617 & $1.239 \mathrm{e}$ & $10.00 f$ & $17.60 \mathrm{c}$ & $3.13 d$ & $15.20 \mathrm{~b}$ & 51.30 & 86.36 & 50.20 & 66.25 \\
\hline 7-Control : Uninfested & $0.00 \mathrm{a}$ & $0.00 \mathrm{a}$ & $1.355 \mathrm{~b}$ & $1.355 \mathrm{~b}$ & $19.60 \mathrm{~b}$ & $19.60 \mathrm{~b}$ & $15.40 \mathrm{~b}$ & $15.40 \mathrm{~b}$ & 78.57 & 78.57 & 0.00 & 0.00 \\
\hline \multicolumn{13}{|c|}{$2006-2007$} \\
\hline 1- Ocimum basilicum & $33.33 \mathrm{c}$ & $2.33 \mathrm{c}$ & $1.020 \mathrm{e}$ & $1.225 \mathrm{~cd}$ & $16.87 \mathrm{c}$ & $18.47 \mathrm{bc}$ & $12.93 d$ & $14.53 \mathrm{cf}$ & 79.81 & 78.67 & 16.73 & 11.07 \\
\hline 2- Syzygium aromaticum L. & $22.22 \mathrm{~b}$ & $1.33 \mathrm{bc}$ & $1.113 \mathrm{c}$ & $1.280 \mathrm{c}$ & $17.33 b$ & $18.80 \mathrm{~b}$ & $14.27 \mathrm{~b}$ & $15.73 b$ & 82.34 & 83.67 & 13.04 & 9.28 \\
\hline 3- Mentha viridis $\mathrm{L}$. & $42.44 \mathrm{~d}$ & $2.67 \mathrm{~cd}$ & $1.030 \mathrm{e}$ & $1.280 \mathrm{c}$ & $16.87 c$ & $19.00 \mathrm{a}$ & $12.80 \mathrm{e}$ & $15.67 \mathrm{bc}$ & 75.87 & 82.47 & 19.53 & 18.32 \\
\hline 4- Cuminum cyminum $\mathrm{L}$. . & $0.00 \mathrm{a}$ & $0.00 \mathrm{a}$ & $1.035 \mathrm{~d}$ & $1.172 \mathrm{~d}$ & $17.00 \mathrm{bc}$ & $17.40 \mathrm{c}$ & $13.20 \mathrm{c}$ & $13.73 d$ & 77.64 & 78.90 & 11.37 & 3.86 \\
\hline 5- Rizolex T-50. & $0.00 \mathrm{a}$ & $0.00 \mathrm{a}$ & $1.399 a$ & $1.435 \mathrm{a}$ & $18.93 a$ & $19.00 \mathrm{a}$ & $15.80 \mathrm{a}$ & $16.47 a$ & 83.56 & 86.68 & 2.50 & 4.06 \\
\hline 6- Control : Infested & 88.89 e & $6.67 d$ & $0.607 \mathrm{k}$ & $1.358 \mathrm{~b}$ & $9.80 \mathrm{~d}$ & $1900 a$ & $6.40 \mathrm{e}$ & $14.73 \mathrm{c}$ & 65.31 & 77.52 & 55.35 & 56.55 \\
\hline 7-Control : Uninfested & $0.00 \mathrm{a}$ & $0.00 \mathrm{a}$ & $1.240 \mathrm{c}$ & $1.240 \mathrm{e}$ & $19.27 \mathrm{~b}$ & $19.27 \mathrm{de}$ & $16.60 \mathrm{~b}$ & $16.60 \mathrm{~d}$ & 26.14 & 86.14 & 0.00 & 0.00 \\
\hline
\end{tabular}

Mean followed by the same letter are not significantly different at the $5 \%$ level by DMRT. 